\title{
Experimental Observation of Laser Control: Electronic Branching in the Photodissociation of $\mathrm{Na}_{2}$
}

\author{
A. Shnitman, ${ }^{1}$ I. Sofer, ${ }^{2}$ I. Golub,${ }^{1}$ A. Yogev, ${ }^{2}$ and M. Shapiro \\ ${ }^{1}$ Department of Chemical Physics, The Weizmann Institute of Science, Rehovot, Israel 76100 \\ ${ }^{2}$ Department of Energy and Environment, The Weizmann Institute of Science, Rehovot, Israel 76100
}

\author{
Z. Chen and P. Brumer \\ Chemical Physics Theory Group and The Ontario Laser and Lightwave Research Centre, University of Toronto, \\ Toronto, Canada M5S 1A1
}

(Received 27 September 1995)

Control over the product branching ratio in the photodissociation of $\mathrm{Na}_{2}$ into $\mathrm{Na}(3 s)+\mathrm{Na}(3 p)$ and $\mathrm{Na}(3 s)+\mathrm{Na}(3 d)$ is demonstrated using a two-photon incoherent interference control scenario. Ordinary pulsed nanosecond lasers are used and $\mathrm{Na}_{2}$ is at thermal equilibrium in a heat pipe. Results show a depletion in the $\mathrm{Na}(3 d)$ product of at least $25 \%$ and a concomitant increase in the $\mathrm{Na}(3 p)$ yield as the relative frequency of the two lasers is scanned.

PACS numbers: $34.50 . \mathrm{Rk}, 33.80 . \mathrm{Gj}$

We report the experimental observation of laser control over a branching photochemical reaction. The reaction studied is the two-photon dissociation of the $\mathrm{Na}_{2}$ molecule at energies where one $\mathrm{Na}$ atom is in its ground state and one $\mathrm{Na}$ atom is in the $3 p, 4 s$, or $3 d$ states, i.e.,

$$
\begin{aligned}
& \mathrm{Na}(3 s)+\mathrm{Na}(3 p), \\
\mathrm{Na}_{2} \stackrel{2 h \nu}{\rightarrow} & \mathrm{Na}(3 s)+\mathrm{Na}(4 s), \\
& \mathrm{Na}(3 s)+\mathrm{Na}(3 d) .
\end{aligned}
$$

Control is demonstrated over the $\mathrm{Na}(3 d) / \mathrm{Na}(3 p)$ branching ratio.

Achieving laser control over dynamical processes has been a long-standing goal of both physicists and chemists. Recent theoretical work [1-3] has shown that this goal may be achieved by manipulating quantum interferences, an area of research known as coherent control. Experimental verification of the basic principles of coherent control have followed [4-12] showing, for example, that total ionization rates can be coherently modulated [6-9] and that current directionality can be phase controlled [7-12]. However, there has only been one very recent report [13] of the primary aim of coherent control: to successfully manipulate integral yields into different competing product channels. Here we present an experimental demonstration of such control.

Our approach is based upon our recent theoretical prediction [14] that laser induced continuum structure (LICS) [15] can give rise to final channel selectivity. In this arrangement one gives structure to the continuum by optically dressing it with a bound state. We showed theoretically [14] that if we dress the continuum with an initially unpopulated bound state using a laser field of frequency $\omega_{2}$, while exciting a populated bound state to this dressed continuum using a laser field of frequency $\omega_{1}$, then a quantum interference arises whose destructive or constructive character depends upon the final channel. (An illustration of this scenario as it applies to $\mathrm{Na}_{2}$ is shown in Fig. 1.) Theoretical studies [14] further showed that the character of this interference depends on the relative frequency between the two light fields, and that selectivity between the $\mathrm{Na}(3 p)$ and $\mathrm{Na}(3 d)$ channels [Eq. (1)] can be achieved by varying $\omega_{1}$ or $\omega_{2}$. This effect is virtually independent of the relative phase between the two light fields; i.e., the light fields need not be coherent. Thus, although the control depends on quantum interference, these interferences are not destroyed by incoherence of the incident laser radiation.

The fact that this control scenario does not require laser coherence makes it especially attractive for laboratory use

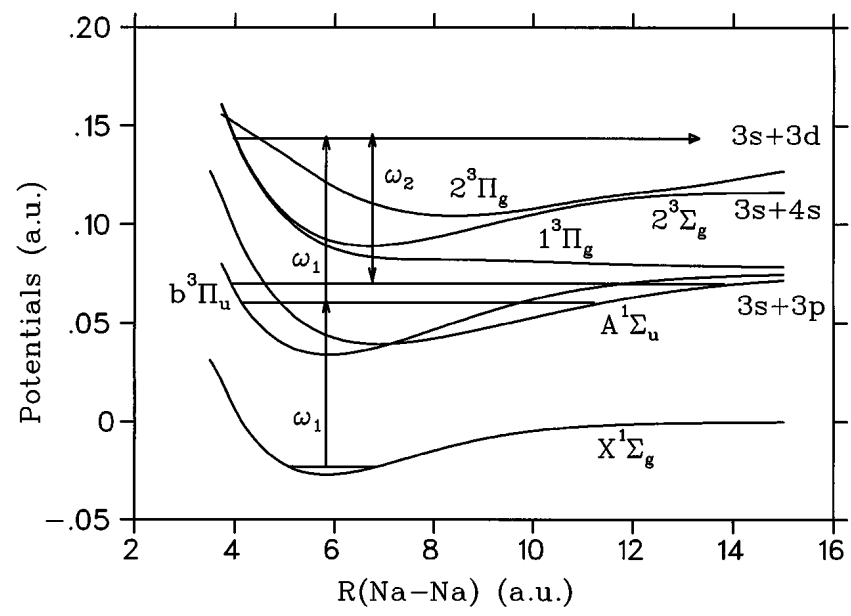

FIG. 1. Incoherent interference control (IIC) scheme and potential energy curves for $\mathrm{Na}_{2}$. In this scheme a $2 \omega_{1}$-photon excitation interferes with an $\omega_{2}$ photon. The two-photon process proceeds from an initial sate, assigned here as $(v=$ $5, J=37$ ), via the $v=35, J=36,38$ levels, belonging to the interacting $A^{1} \Sigma_{u} /{ }^{3} \Pi_{u}$ electronic states, acting as intermediate resonances. The $\omega_{2}$ photon dresses the continuum with the (initially unpopulated) $v=93, J=36$ and $v=93, J=38$ levels of the $A^{1} \Sigma_{u} /{ }^{3} \Pi_{u}$ electronic states. 
since generally available, nontransform limited, nsec dye lasers can be used. In our experiment we use two dye lasers pumped by a frequency-doubled Nd-YAG laser. One dye laser, whose frequency $\omega_{2}$ was tuned between 13312 and $13328 \mathrm{~cm}^{-1}$, was used to dress the continuum with a vibrotational state of the $A^{1} \Sigma_{u} /{ }^{3} \Pi_{u}$ mixed electronic state [16] of $\mathrm{Na}_{2}$. The other dye laser, whose frequency $\omega_{1}$ was fixed at $17474.12 \mathrm{~cm}^{-1}$, was used to induce a two-photon dissociation of the $v=5, J=37$ ground state of $\mathrm{Na}_{2}$, through intermediate resonances (assigned as $v=35, J=38$ and $v=35, J=36$ ) of the $A^{1} \Sigma_{u} /{ }^{3} \Pi_{u}$ mixed state. Our $\omega_{1}$ and $\omega_{2}$ pulses, both of $\sim 5 \mathrm{nsec}$ duration with the stronger amongst them $\left(\omega_{2}\right)$ having an energy of $\sim 3.5 \mathrm{~mJ}$, were made to overlap in a heat pipe containing $\mathrm{Na}$ vapor at $370-410{ }^{\circ} \mathrm{C}$. Spontaneous emission from the excited $\mathrm{Na}$ atoms $[\mathrm{Na}(3 d) \rightarrow$ $\mathrm{Na}(3 p)$ and $\mathrm{Na}(3 p) \rightarrow \mathrm{Na}(3 s)]$ resulting from the $\mathrm{Na}_{2}$ photodissociation was detected and dispersed in a spectrometer and a detector with a narrow bandpass filter.

Figure 2 shows experimental $\mathrm{Na}(3 d)$ and $\mathrm{Na}(3 p)$ emission as a function of $\omega_{2}$ at a fixed $\omega_{1}$. Each point represents an average over a few hundred laser shots, each chosen to have an $\omega_{2}$ pulse energy which deviates by less than $5 \%$ from $3.5 \mathrm{~mJ}$. At these energies we estimate our pulse intensity to be $\sim 10^{7} \mathrm{~W} / \mathrm{cm}^{2}$. We see that when the $\mathrm{Na}(3 d)$ yield dips, the $\mathrm{Na}(3 p)$ yield peaks, in accordance with theoretical expectation [17]. The controlled modulation of the $\mathrm{Na}(3 p) / \mathrm{Na}(3 d)$ branching ratio is seen to exceed $30 \%$.

The theoretical calculations [17] of the $\mathrm{Na}(3 d)$ yield resulting from photodissociation of a single initial $\mathrm{Na}_{2}$ bound state are presented in Fig. 3 and contrasted with the experimental results of Fig. 2. The same is done in Fig. 4 for the $\mathrm{Na}(3 p)$ yield. We see two major $\mathrm{Na}(3 d)$ dips, accompanied by $\mathrm{Na}(3 p)$ peaks, in good

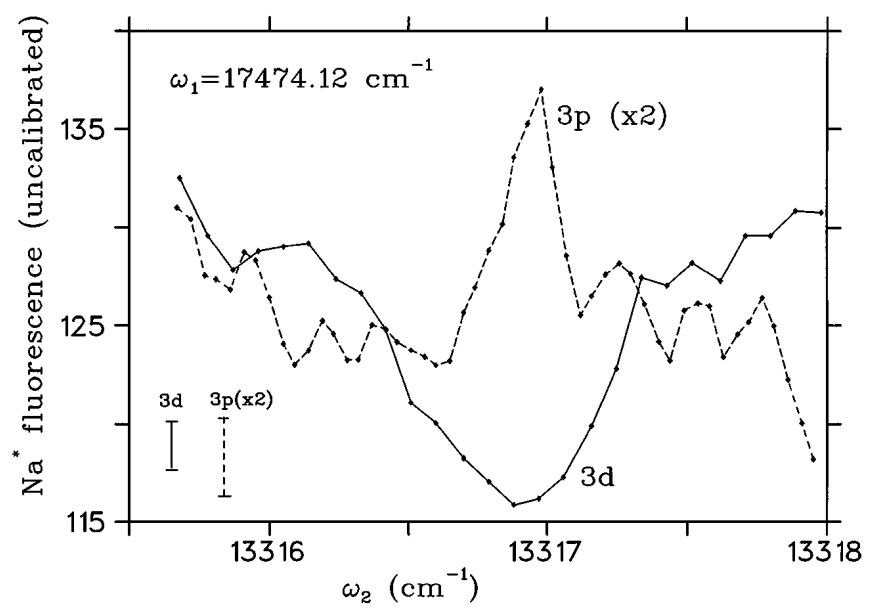

FIG. 2. Experimental $\mathrm{Na}(3 d)$ fluorescence (solid) and $\mathrm{Na}(3 p)$ fluorescence (dashed) (both uncalibrated) for the $\mathrm{Na}_{2} \rightarrow \mathrm{Na}(3 s)+\mathrm{Na}(3 d), \mathrm{Na}(3 p)$ IIC scenario whose details are given in Fig. 1, as a function of the $\omega_{2}$ frequency. The $\omega_{1}$ frequency is fixed at $17474.12 \mathrm{~cm}^{-1}$. agreement with the experiment. The calculations were done for the initially unpopulated $v=93, J=31$ and $v=93, J=33$ levels of the mixed $A^{1} \Sigma_{u} /{ }^{3} \Pi_{u}$ electronic state, accessed via the $v=33, J=31$ and $v=33, J=$ 33 intermediate resonances by the $2 \omega_{1}$-photon process. These $v, J$ values differ slightly from those which we experimentally assigned ( $v=35$ and $J=36$ and 38), but the line shapes were found to change very little with small changes in $v, J$. Considering the uncertainties in the theoretical potentials used [17,18], the agreement between theory and experiment [especially in the $\mathrm{Na}(3 d)$ signal] is impressive. Additional computations [14] suggest that the observed experimental substructures may be due to the excitation of numerous additional, as yet unassigned, thermally populated vibrotational $\mathrm{Na}_{2}$ energy levels.

The $\mathrm{Na}(3 p)$ experimental signal is superimposed on a high background due to population of the $\mathrm{Na}(3 p)$ state by emission from the $\mathrm{Na}(3 d)$ and $\mathrm{Na}(4 s)$ states, and due to direct population of $\mathrm{Na}(3 p)$ from the $\mathrm{Na}_{2}$ molecule by an $\omega_{1}+\omega_{2}$ absorption [not possible energetically for the $\mathrm{Na}(3 d)$ channel]. We also had to overcome radiation trapping effects by monitoring the $\mathrm{Na}(3 p) \rightarrow \mathrm{Na}(4 s)$ emission off line center. The results shown in Figs. 2 and 4 are obtained by subtracting the contribution of these processes from the observed $\mathrm{Na}(3 p)$ signal. To do so we calibrated the contribution from the $\mathrm{Na}(3 d) \rightarrow \mathrm{Na}(3 p)$ emission via a separate experiment, where we monitored the $\mathrm{Na}(3 p)$ signal resulting from the direct two-photon excitation of the $\mathrm{Na}(3 d)$ state. The direct $\omega_{1}+\omega_{2}$ contribution was accounted for by measuring the $\mathrm{Na}(3 p)$ signal at different $\omega_{1}+\omega_{2}$ intensities. Because we saturate the one-photon $\omega_{1}$ resonance, the dependence

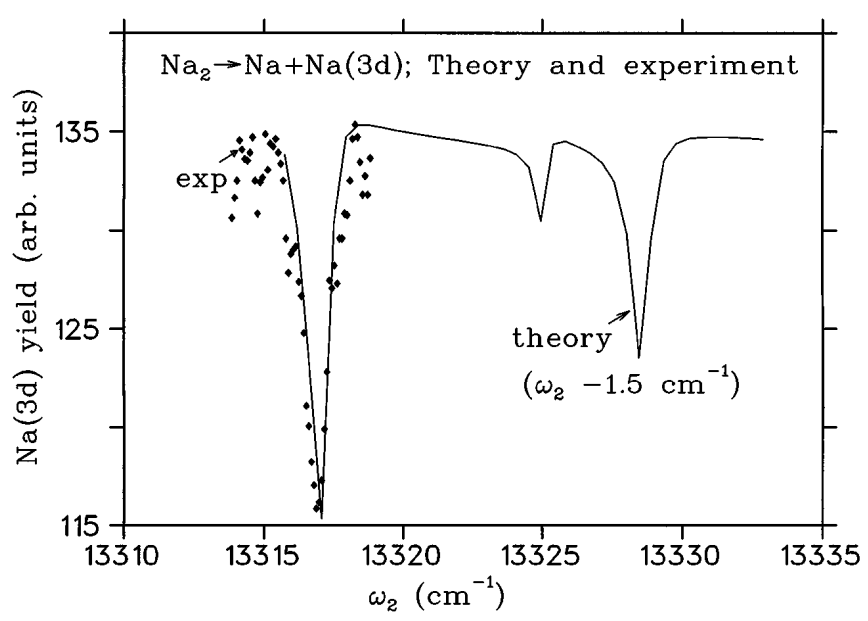

FIG. 3. Comparison of the experimental and theoretical $\mathrm{Na}_{2} \rightarrow \mathrm{Na}(3 s)+\mathrm{Na}(3 d)$ yields as a function of $\omega_{2}$. In the calculation, an intermediate $v=33, J=31,33$ resonance is used and $\omega_{1}$ is fixed at $17720 \mathrm{~cm}^{-1}$. The intensities of the two laser fields are $I\left(\omega_{1}\right)=1.72 \times 10^{8} \mathrm{~W} / \mathrm{cm}^{2}$ and $I\left(\omega_{2}\right)=2.84 \times 10^{8} \mathrm{~W} / \mathrm{cm}^{2}$. The $\omega_{2}$ frequency axis of the calculated results was shifted by $-1.5 \mathrm{~cm}^{-1}$ in order to better compare the predicted and measured line shapes. 


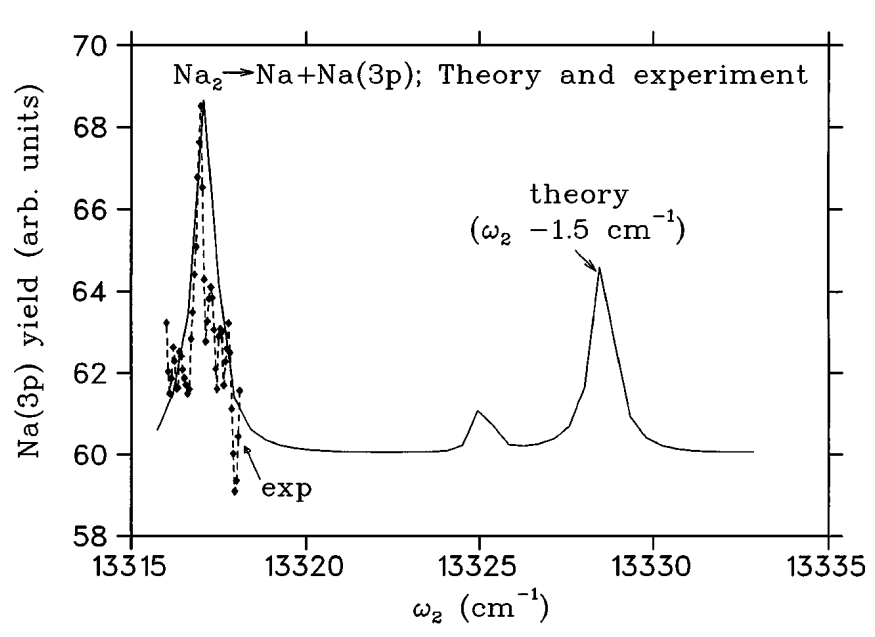

FIG. 4. Comparison of the experimental and theoretical $\mathrm{Na}_{2} \rightarrow \mathrm{Na}(3 s)+\mathrm{Na}(3 p)$ yields as a function of $\omega_{2}$, with parameters as in Fig. 3.

of the $\omega_{1}+\omega_{2}$ process was found to be linear in the $\omega_{2}$ intensity. Hence, determining the slope and intercept of this linear dependence allowed us to subtract out its contribution at the experimental $\omega_{1}$ and $\omega_{2}$ intensities.

To confirm that the observed $\mathrm{Na}(3 d)$ dip and $\mathrm{Na}(3 p)$ peak structures are indeed due to incoherent interference control, i.e., the interference between the $\omega_{1}$ and $\omega_{2}$ induced optical processes, we ran the following checks.

(1) We verified that what we are seeing is a strong field effect by changing the $\omega_{2}$ power. Reducing the power by a factor of $\sim 50$ resulted in the complete vanishing of the dip and peak structures.

(2) We verified that the observed structures are due to the combined action of the two lasers by delaying the $\omega_{1}$ pulse relative to the $\omega_{2}$ pulse. A delay of $\pm 19 \mathrm{nsec}$, guaranteeing no overlap between the pulses, completely eliminated the $\mathrm{Na}(3 d)$ dips.

(3) We rotated the planes of polarization of the two lasers and noted no change in the observed $\mathrm{Na}^{*}$ fluorescence. This indicates that the observed peaks and valleys are not due to the polarization of the sodium atoms. It also demonstrates that the $\mathrm{Na}$ atoms resulting from the dissociation are unpolarized.

(4) Real time measurements of the rise and decay of the $\mathrm{Na}(3 d)$ and $\mathrm{Na}(3 p)$ signals were performed. If the observed structures are due to an accidental secondary transfer of population from the $\mathrm{Na}(3 d)$ to the $\mathrm{Na}(3 p)$ state then such a mechanism would be reflected in the time dependence of the $\mathrm{Na}(3 d)$ signal. Specifically, if a (collisional or other relaxational) mechanism was in effect at one $\omega_{2}$ frequency and not at another, thus giving rise to the observed $\mathrm{Na}(3 d)$ dip and $\mathrm{Na}(3 p)$ peak at that particular $\omega_{2}$ frequency, we would see a faster decay of the $\mathrm{Na}(3 d)$ signal at that frequency relative to the other. Our findings show an identical decay curve for the $\mathrm{Na}(3 d)$ signal at all $\omega_{2}$ frequencies probed. The only thing which changes is the area under the decay curve. This indicates that it is the actual production of the $\mathrm{Na}(3 d)[\mathrm{Na}(3 p)]$ state which is affected by changing $\omega_{2}$, and not its subsequent decay [buildup].

(5) We shifted the $\omega_{1}$ frequency (by -0.19 and $-0.40 \mathrm{~cm}^{-1}$ ) and examined the effect of such shifts on the dependence of the $\mathrm{Na}(3 d)$ and $\mathrm{Na}(3 p)$ yields on $\omega_{2}$. Since the two-photon $\omega_{1}$ absorption is mediated by a (saturated) intermediate one-photon resonance, the $\omega_{2}$ dependence of the $\mathrm{Na}(3 d)$ and $\mathrm{Na}(3 p)$ structures should move by an amount equal to the $\omega_{1}$ shift. This is indeed the case, as demonstrated in Fig. 5, where the $\omega_{2}$-dependent structures are seen to redshift, respectively, by 0.23 and $0.37 \mathrm{~cm}^{-1}$. These values are, within our frequency resolution of $\pm 0.04 \mathrm{~cm}^{-1}$, in perfect accord with the above expectations. A similar shift of the $\mathrm{Na}(3 p)$ peaks was also observed, thus verifying the optical origin of the effect.

In summary, we have experimentally demonstrated laser control of branching photochemical reactions using quantum interference phenomena. In addition, we have overcome two major experimental obstacles to the general implementation of optical control of reactions: (a) we have achieved control using incoherently related light sources, and (b) we have affected control in a bulk, thermally equilibrated, system.

We wish to acknowledge the invaluable help of I. Levy and Z. Kotler in building the experiment. This work was supported by the Minerva Foundation, Germany, by the Israel Academy of Sciences Equipment Programme and

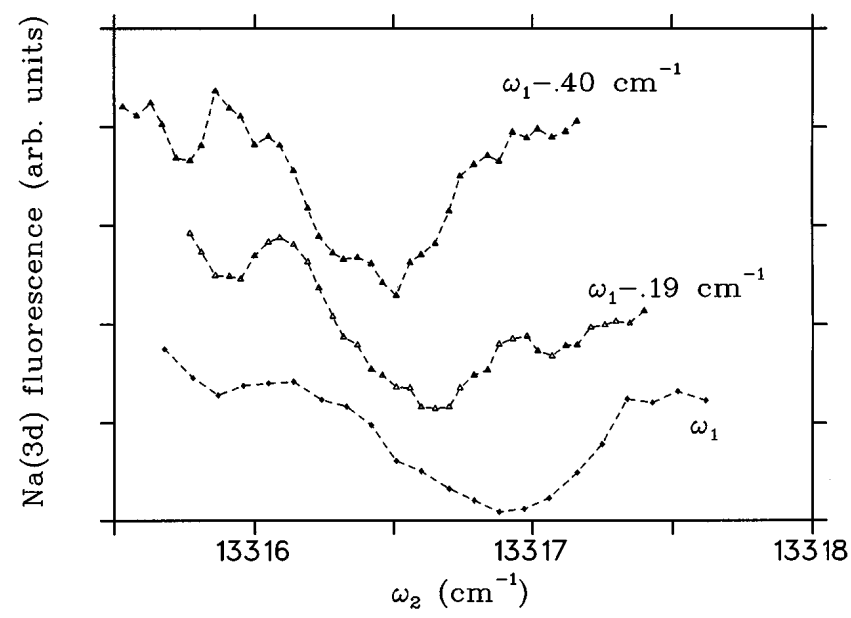

FIG. 5. The $\mathrm{Na}(3 d)$ fluorescence as a function of $\omega_{2}$ for three different $\omega_{1}$ frequencies. The lowest trace corresponds to an $\omega_{1}$ value of $17474.12 \mathrm{~cm}^{-1}$. The upper traces result from redshifting the $\omega_{1}$ frequency by 0.19 and $0.40 \mathrm{~cm}^{-1}$. We observe a redshift in the $\omega_{2}$ dependence of the $\mathrm{Na}(3 d)$ yield of, respectively, 0.23 and $0.37 \mathrm{~cm}^{-1}$, each being, without experimental uncertainty of $\pm 0.04 \mathrm{~cm}^{-1}$, identical to the respective $\omega_{1}$ shift. Note that the other substructures of the $\mathrm{Na}(3 d)$ line shapes show similar shifts. 
by the U.S. Office of Naval Research under Contract No. N00014-90-J-1014.

[1] For recent reviews, see M. Shapiro and P. Brumer, Int. Rev. Phys. Chem. 13, 187 (1994); P. Brumer and M. Shapiro, Annu. Rev. Phys. Chem. 43, 257 (1992). For the initial paper, see P. Brumer and M. Shapiro, Chem. Phys. Lett. 126, 541 (1986).

[2] See D. J. Tannor and S. A. Rice, Adv. Chem. Phys. 70, 441 (1988); R. Kosloff, S. A. Rice, P. Gaspard, S. Tersigni, and D. J. Tannor, Chem. Phys. 139, 201 (1989); S. Shi and H. Rabitz, Chem. Phys. 139, 185 (1989); Y. Yan, R. E. Gillilan, R. M. Whitnell, and K. R. Wilson, J. Phys. Chem. 97, 2320 (1993).

[3] A. D. Bandrauk, J.-M. Gauthier, and J.F. McCann, Chem. Phys. Lett. 200, 399 (1992); M. Yu. Ivanov, P. B. Corkum, and P. Dietrich, Laser Phys. 3, 375 (1993).

[4] C. Chen, Y-Y. Yin, and D. S. Elliott, Phys. Rev. Lett. 64, 507 (1990); 65, 1737 (1990).

[5] S. M. Park, S-P. Lu, and R. J. Gordon, J. Chem. Phys. 94, 8622 (1991); S-P. Lu, S. M. Park, Y. Xie, and R. J. Gordon, J. Chem. Phys. 96, 6613 (1992).

[6] V.D. Kleiman, L. Zhu, X. Li, and R. G. Gordon, J. Chem. Phys. 102, 5863 (1995).

[7] G. Kurizki, M. Shapiro, and P. Brumer, Phys. Rev. B 39, 3435 (1989).
[8] B. A. Baranova, A. N. Chudinov, and B. Ya Zel'dovitch, Opt. Commun. 79, 116 (1990).

[9] Y-Y. Yin, C. Chen, D. S. Elliott, and A. V. Smith, Phys. Rev. Lett. 69, 2353 (1992).

[10] E. Dupont, P. B. Corkum, H. C. Liu, M. Buchanan, and Z. R. Wasilewski, Phys. Rev. Lett. 74, 3596 (1995).

[11] B. Sheeny, B. Walker, and L. F. Dimauro, Phys. Rev. Lett. 74, 4799 (1995).

[12] Y.-Y. Yin, R. Shehadeh, D. Elliott, and E. Grant, Chem. Phys. Lett. 241, 591 (1995).

[13] L. Zhu, V. Kleiman, X. Li, S.P. Lu, K. Trentelman, and R. J. Gordon (to be published).

[14] Z. Chen, M. Shapiro, and P. Brumer, Chem. Phys. Lett. 228, 289 (1994); J. Chem. Phys. 102, 5683 (1995); Phys. Rev. A 52, 2225 (1995).

[15] See, for example, P.L. Knight, M. A. Lauder, and B. J. Dalton, Phys. Rep. 190, 1 (1990), and references therein; O. Faucher, D. Charalambidis, C. Fotakis, J. Zhang, and P. Lambropoulos, Phys. Rev. Lett. 70, 3004 (1993)

[16] Z. Chen, M. Shapiro, and P. Brumer, J. Chem. Phys. 98, 8647 (1993); 98, 6843 (1993).

[17] Theoretical calculations reported here are for cw excitation. Pulsed laser based computational studies are ongoing [Z. Chen, P. Brumer, and M. Shapiro (unpublished)].

[18] The potential curves and the relevant electronic dipole moments are from I. Schmidt, Ph.D. thesis, Kaiserslautern University, 1987. 